\title{
FIRST FATIGUE MEASUREMENTS ON THICK EPI-POLYSILICON MEMS IN ULTRA-CLEAN ENVIRONMENT
}

\author{
Anne L. Alter ${ }^{1 *}$, Ian B. Flader ${ }^{1}$, Yunhan Chen ${ }^{2}$, Lizmarie Comenencia Ortiz ${ }^{1}$, \\ Dongsuk D. Shin ${ }^{1}$, David B. Heinz ${ }^{1}$, and Thomas W. Kenny ${ }^{l}$ \\ ${ }^{1}$ Stanford University, Stanford, California, USA \\ ${ }^{2}$ Apple Inc., Cupertino, California, USA
}

\begin{abstract}
This paper studies, for the first time, fatigue in vacuum encapsulated, oxide-free, thick epi-polysilicon MEMS devices. Thick epi-polysilicon is the device layer material used in more than 1B MEMS inertial sensors produced for consumer electronics and automotive safety system applications every year. The ultra-clean encapsulation process eliminates the sources of environmental effects and prevents formation of surface oxide. In these experiments, we observed fatigue failure in the absence of environmental mechanisms that have typically been linked to fatigue. These results are important due to the ongoing debate among researchers about the mechanisms for fatigue failure in micro-scale silicon MEMS devices.
\end{abstract}

\section{INTRODUCTION}

\section{Background}

Fatigue in MEMS resonators is a primary concern when considering the long-term performance and reliability of devices particularly those subject to cyclic loading, such as Coriolis gyroscopes [1]. Gyroscopes and other inertial sensors usually require high amplitude responses, which can lead to stress concentrations within their surfaces. Material failure due to fatigue occurs at stresses much lower than the fracture strength [2], so there is potential to for devices to fail under typical operating conditions.

Even though silicon is a brittle material that does not exhibit dislocation activity at room temperature and thus should not fatigue at room temperature [3], fatigue has been observed in many studies of micrometer-sized silicon. The mechanisms behind micrometersized silicon fatigue failure remain controversial due to difficulties in isolating and controlling all the environmental conditions that could contribute to fatigue onset.

Past research has focused on fatigue mechanisms arising from effects taking place in surficial oxides [4][5][6], and is typically studied in environments where the presence of oxygen and relative humidity are factors. The primary fatigue mechanisms that have arisen from this research are 'reaction-layer' fatigue proposed by Muhlstein et al., which requires oxidation of exposed silicon surfaces [5], and the 'subcritical cracking' model proposed by Kahn, in which surface debris, oxide, or other deformities create a wedging effect in the native silicon [6].

Fatigue has been studied in ultra-clean, encapsulated, inert environments, where relative humidity is not a factor for both smooth-walled single crystal silicon (SCS) [7][8] and oxide-coated, rough-walled SCS devices [8]. Results showed no fatigue in SCS up to $7.5 \mathrm{GPa}$, a threshold twice as high as other research efforts were able to achieve. The absence of fatigue was attributed to the ultraclean encapsulated environment, and hydrogen smoothed surfaces, and aligns with theories that propose that bulk silicon should not fatigue at room temperature. The oxide-coated SCS had a reduced threshold of $2.7 \mathrm{GPa}$, which was attributed to the subcritical cracking model and discussed that introducing oxide to the environment accelerated the fatigue life of these structures by locking in surface structural defects [8].

Polycrystalline silicon (polysilicon), an amorphous form of silicon consisting of random grain boundaries and crystal orientations, has been shown to fatigue at the micrometer scale $[4][5][6][9][10][11][12][13]$. The polysilicon structures that have been studied are thin-films, typically $<10 \mu \mathrm{m}$, fabricated using LPCVD processes, and are coated in oxide. The structures that have fatigued have occurred in imperfect environmental conditions, although some oxide-coated polysilicon devices have shown fatigue resistance in high vacuum [10].

This work reports the first study of fatigue in vacuum encapsulated, ultra-clean, thick $(40 \mu \mathrm{m})$ epi-polysilicon structures, whose surfaces are free of oxide, and where the inert environment prevents the formation of additional surface oxide. These unique environmental conditions enable the study of intrinsic material fatigue behavior of polysilicon and is the first study of fatigue in thick epi-polysilicon, which is the device layer material used in more than 1B MEMS inertial sensors produced for consumer electronics and automotive safety system applications every year.

\section{EXPERIMENT METHODS}

\section{Device Design and Experiment Setup}

As shown in Fig. 1, a MEMS device, consisting of a Y-shaped epi-polysilicon proof mass, was biased to a DC voltage and was electrostatically actuated and sensed by a time-varying AC signal applied to the combs on the perimeter. The proof mass was attached to a notched cantilever beam, which is a point of high stress concentration and dictates the ultimate compliance of the structure. The device was driven into resonance, the signal amplified by a trans-impedance amplifier (TIA), and a phase-locked loop (PLL) was used to oscillate the device and track the frequency over time.

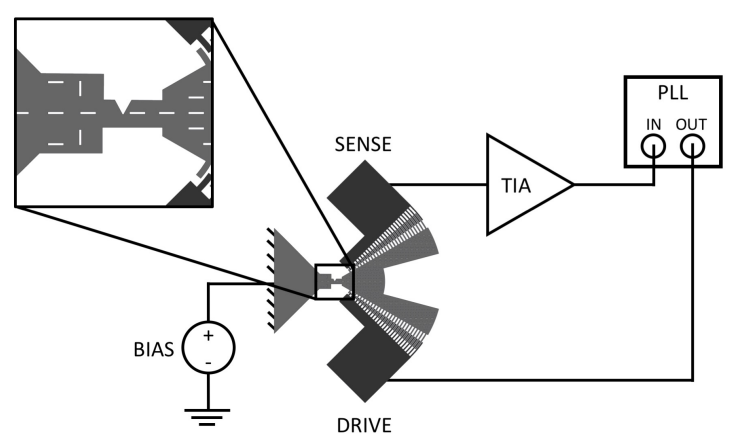

Figure 1: Y-shaped fatigue structure, which is electrostatically driven and sensed, and oscillated in a phase-locked loop. Inset shows notch geometry.

\section{Device Fabrication}

The epi-polysilicon fatigue structure was fabricated in an ultraclean, vacuum-encapsulated package. The fabrication process [14] was derived from a large-gap SCS encapsulation process [15] and a process that incorporated a polysilicon device layer to the large gap process [16]. The device layer, a $40 \mu \mathrm{m}$ thick, N-type polysilicon film, was epitaxially deposited. During the fabrication, all oxide that had been surrounding the device layer was etched with a vapor- 
phase hydrofluoric acid, releasing the device structure and leaving the device layer surfaces oxide-free. A final high temperature annealing step during device sealing caused the surfaces to roughen due to silicon migration on the randomly oriented epi-polysilicon grain structure [17]. The fabricated device structure and surface roughness are shown in Fig. 2. The surface roughness is $<0.3 \mu \mathrm{m}$ which is much smaller than the notch size of just under $7 \mu \mathrm{m}$ deep.

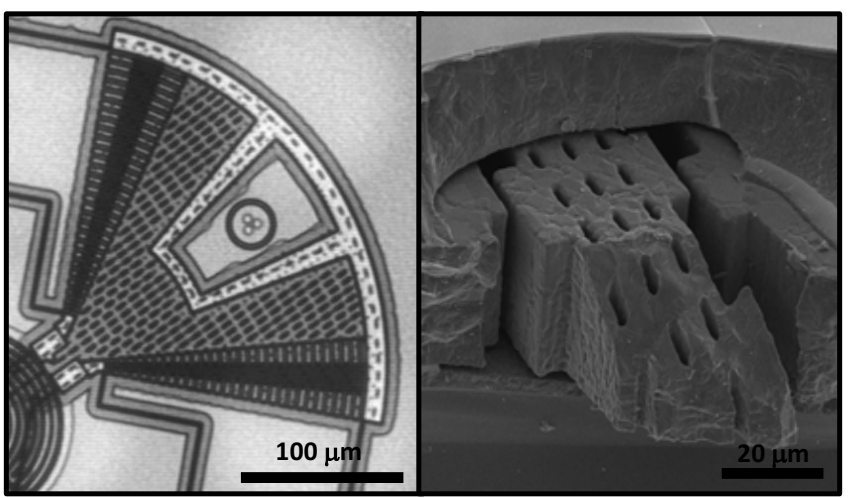

Figure 2: Infrared image of fatigue structure (left); SEM showing surface roughness of epi-polysilicon structure (right).

\section{Experimental Procedure}

Devices were biased with a constant $10 \mathrm{~V}$ DC signal and initially driven with a low amplitude AC signal through the comb drive structures. The initial resonant frequency and phase condition were determined and a PLL was used to lock into the signal and maintain the phase condition over time. The frequency stability, a measure of device compliance and thus notch stability, was measured over 20-hour intervals. After each 20-hour interval, the drive amplitudes were increased (incremented by $50 \mathrm{mV}$ ), thus increasing the stress at the notch, and the stability measurement was repeated. This process of increasing the AC drive voltage after 20hour intervals was repeated until device failure.

\section{RESULTS}

Fatigue failure was observed in four epi-polysilicon devices and their results are summarized in the following figures. The figures show differing failure drift profiles, along with differing rates of accelerated frequency drift to failure. Imaging results confirm that device failure occurred due to cracking at the notch region, and when plotting results on a $\mathrm{S}-\mathrm{N}$ curve, we validate that they fit into trend of other similar device fatigue failures.

\section{Frequency Drift Characteristics}

As shown in Fig. 3, 'Device 1' showed stability for approximately 161.9 hours. The device was stable at a $650 \mathrm{mV}$ drive amplitude for about 1.9 hours before it suddenly began to drift in frequency at a linear accelerated rate. The device drifted approximately $18 \mathrm{ppm}$ before failing at approximately 162.5 hours. The calculated stress at failure, based on the output device amplitude, was $2.3 \mathrm{GPa}$.

As shown in Fig. 4, 'Device 2' showed stability for approximately 20 hours. Upon increasing the AC drive voltage to $350 \mathrm{mV}$ at 20 hours, the frequency drifted quickly, then slowed but continued to drift for about 1 hour. After approximately 21.8 hours, the frequency drift rapidly accelerated, and the device failed at 21.9 hours. The device frequency drifted by a total of approximately $22 \mathrm{ppm}$. The stress at failure was calculated to be $3.5 \mathrm{GPa}$.

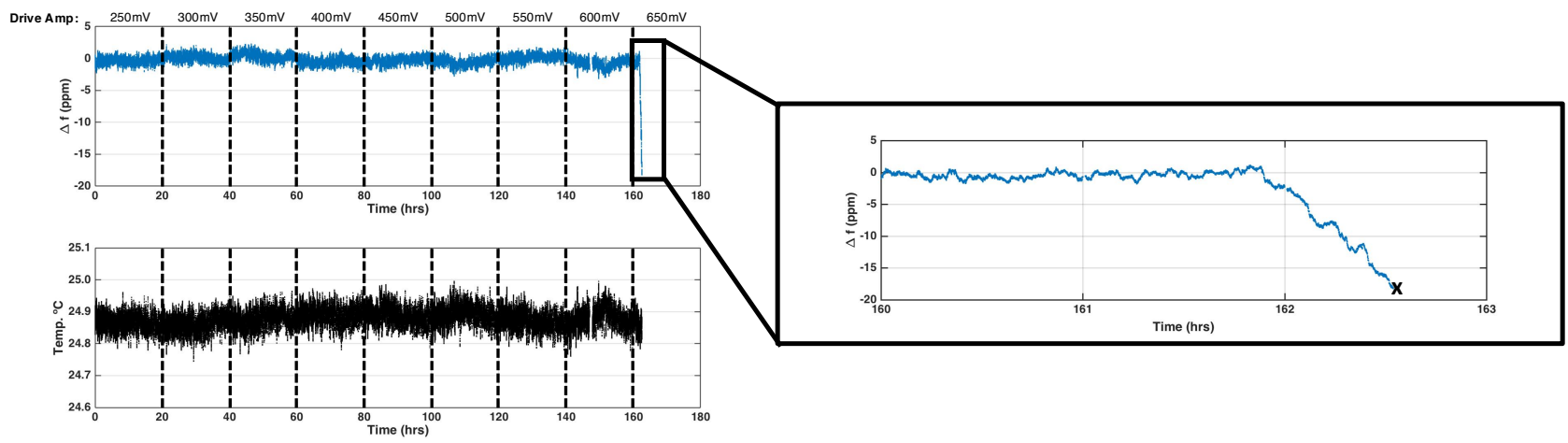

Figure 3: Epi-polysilicon 'Device 1' frequency change and measured temperature over time. Device failure (indicated with an 'x') occurs at 162.5 hours. The drive amplitude was increased at 20-hour intervals as shown at the top of the frequency plot.

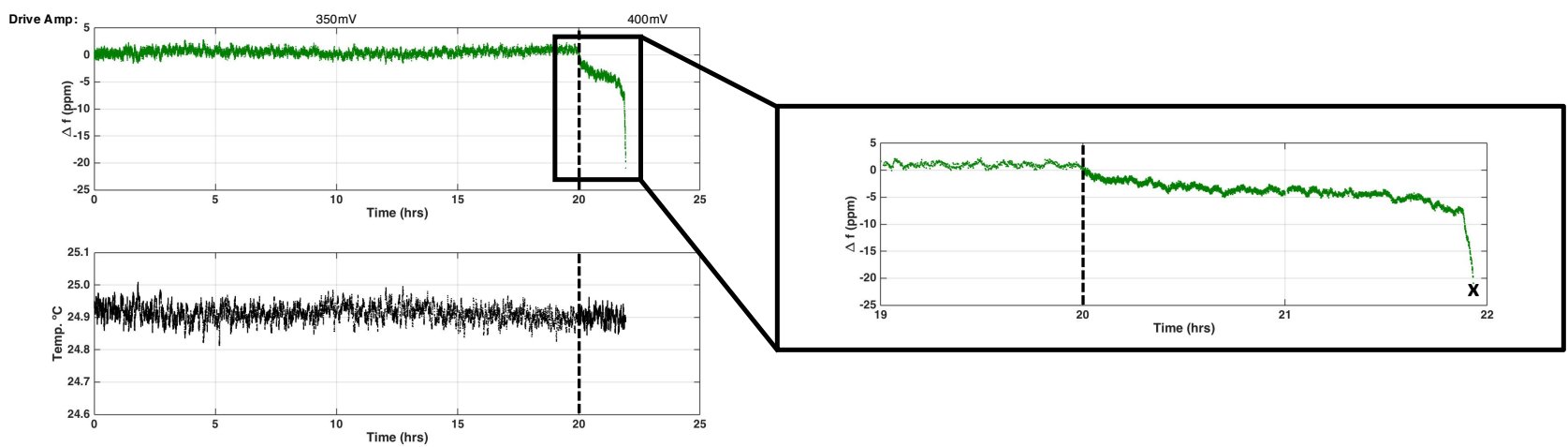

Figure 4: Epi-polysilicon 'Device 2' frequency change and measured temperature over time. Device failure (indicated with an ' $x$ ') occurs at 21.9 hours. The drive amplitude was increased at 20-hour intervals as shown at the top of the frequency plot. 
Before failure, both devices showed stability of $\pm 3 \mathrm{ppm}$ frequency shift. These small shifts in frequency during stability were directly correlated to slight shifts in temperature. The temperature of both devices was maintained at just below $25^{\circ} \mathrm{C}$.

\section{Localized High Resolution Imaging}

Since the device layer is fully encapsulated, there is no way of knowing exactly how the devices failed using typical microscopy techniques. So, X-ray imaging, with nanoscale resolution, was performed, as shown in Fig. 5. The images reveal that the two epi-polysilicon devices, 'Device 1' and 'Device 2', did in fact fracture at the notch region. Since the devices failed at the notch region, the decrease in compliance of the structures can be attributed to cracking at the notch region.
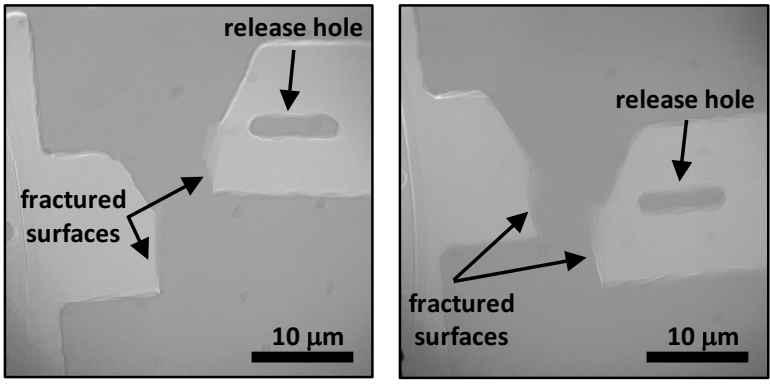

Figure 5: X-ray imaging with nanoscale resolution of fractured notches in epi-polysilicon fatigue 'Device 1' (left) and 'Device 2' (right).

Fig. 5 also reveals some interesting characteristics of device failure. The 'Device 1' crack appears to have initiated directly at the notch tip, while 'Device 2' seems to have propagated just to the left of the notch tip. The images also reveal the rough epipolysilicon side walls. Release holes, which help ensure that all of the surrounding oxide is etched to release the structure in fabrication are also visible.

\section{Accelerated Drift to Failure}

Fig. 6 compares the period just before failure of linear accelerated frequency drift of 'Device 1', 'Device 2', and 2 and two

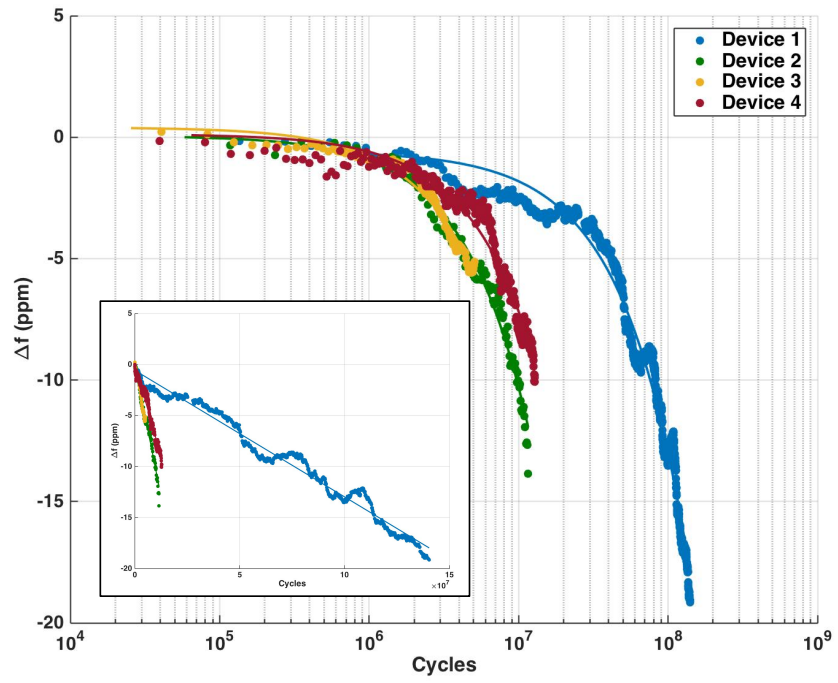

Figure 6: Accelerated drift rate to failure of 4 epi-polysilicon devices. Inset shows same plot on linear scale. other devices of the same geometry. The results are plotted on a semi-log scale with the inset showing a linear scale to accentuate the linear nature of the drift profiles. 'Device 1' takes noticeably longer time to drift to failure. All devices drift between 6 and $22 \mathrm{ppm}$ before failure.

\section{Stress Life Plot}

Fig. 7 summarizes the stress-life fatigue behavior of this work compared with other work on MEMS polysilicon and encapsulated oxide-coated SCS structures. Previous fatigue research in MEMS has shown a 'metal-like' stress-life fatigue behavior for polysilicon thin films and $20 \mu \mathrm{m}$ thick films with increased stresses leading to shorter lifespans and vice versa [5][13]. The thick, $40 \mu \mathrm{m}$, structures in this work seems to fall into this same category of behavior with lower stressed devices having a longer fatigue life than devices exposed to higher stresses. More data points would need to be collected to find a conclusive pattern in the data.

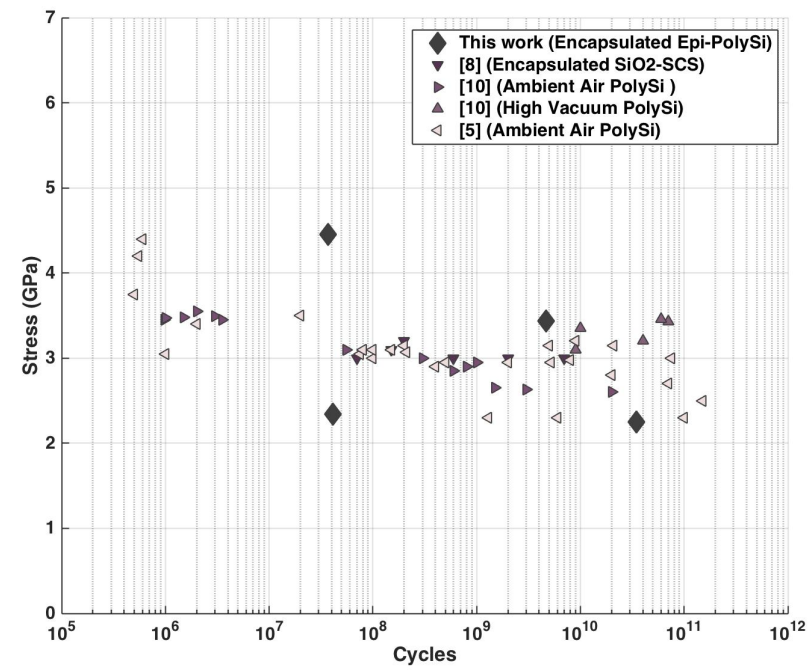

Figure 7: Stress-Life (S-N) plot of this work compared with literature [5][8][10]. This work fits with the trend of lower stress leading to longer fatigue life of the devices and vice versa.

\section{DISCUSSION / CONCLUSIONS}

In conclusion, four epi-polysilicon devices operate up to $2 \mathrm{GPa}$ without fatigue failure, which is consistent with prior observations in less-pristine environments, clearly indicating that previously-postulated environmental causes for fatigue failure in epi-polysilicon are not important. Furthermore, without the presence of oxygen or native debris, the 'reaction-layer' fatigue model is not consistent with these results.

The most plausible cause for fatigue in these structures aligns most closely with the 'subcritical cracking' model proposed by Kahn et al. due, in this case, to the surface roughness of the structures. During compressive cycles, the rough surfaces at the notch tip wedge to create areas of tension, thus propagating irreversible micro-cracks through the structure [6].

The difference in fatigue drift characteristics between 'Device 1' and 'Device 2' could be attributed to randomness in the orientation of the grain structure. 'Device 1', showed unstable frequency drift to failure and likely reached the critical crack length upon crack initiation. In contrast, 'Device 2' showed behavior that indicated crack initiation, followed quickly by crack arrest, and then crack re-initiation just before failure. 'Device 1' showed behavior consistent with continued material weakening upon reaching critical crack length, while 'Device 2' showed signs 
of strengthening during crack arrest, which could be attributed to crack-tip shielding or grain boundary plasticity [18]. Given that all devices do not respond to the drive conditions with the same stress amplitude, due to the randomness in the grain structure, devices may fail by pure weakening or by a combination of weakening and strengthening at the crack tip.

The fatigue failure at the notch tip was validated with X-ray imaging that revealed the devices failed at or close to the notch tip. Furthermore, the results were validated by the stress life behavior of other similar polysilicon devices and encapsulated oxide coated SCS devices.

This first study on thick, $40 \mu \mathrm{m}$, epi-polysilicon encapsulated structures demonstrates fatigue failure as a potential failure mechanism, and should be taken into consideration in future MEMS design.

\section{ACKNOWLEDGEMENTS}

This work was supported by the Defense Advanced Research Projects Agency (DARPA) grant "Precise Robust Inertial Guidance for Munitions (PRIGM)", managed by Dr. Ron Polcawich, Contract Number \# N66001-16-1-4023. This work was performed in part at the Stanford Nanofabrication Facility (SNF), supported by the National Science Foundation under Grand ECS- 9731293. Special thanks to all the staff at SNF for their help during the fabrication process.

\section{REFERENCES}

[1] A.D. Romig Jr, M.T. Dugger, and P.J. McWhorter, "Materials Issues in Microelectromechanical Devices: Science, Engineering, Manufacturability and Reliability", Acta Materialia, 51, 19, (2003).

[2] R.W. Hertzberg, Deformation and Fracture Mechanics of Engineering Materials. 1989.

[3] K. Sumino, "Deformation Behavior of Silicon", Metallurgical and Materials Transactions A, 30, 6, (1999).

[4] S.M. Allameh, P. Shrotriya, A. Butterwick, S.B. Brown, and W.O. Soboyejo, "Surface Topography Evolution and Fatigue Fracture in Polysilicon MEMS Structures", Journal of Microelectromechanical Systems, 12, 3, (2003).

[5] C.L. Muhlstein, E.A. Stach, and R.O. Ritchie, "A ReactionLayer Mechanism for the Delayed Failure of Micron-Scale Polycrystalline Silicon Structural Films Subjected to HighCycle Fatigue Loading", Acta Materialia, 50, 14, (2002).

[6] H. Kahn, R. Ballarini, J.J. Bellante, and A.H. Heuer, "Fatigue Failure in Polysilicon Not Due to Simple Stress Corrosion Cracking", Science, 298, 5596, (2002).

[7] S. Yoneoka, Y.Q. Qu, S. Wang, M.W. Messana, A.B. Graham, J. Salvia, K. Bongsang, R. Melamud, G. Bahl, and T.W. Kenny, "High-Cyclic Fatigue Experiments of Single Crystal Silicon in an Oxygen-Free Environment", IEEE 23rd International Conference on Micro Electro Mechanical Systems (MEMS), Hong Kong, China, 1/24 - 28/10, IEEE, (2010), pp. 224 - 227.

[8] V.A. Hong, S. Yoneoka, M.W. Messana, A.B. Graham, J.C. Salvia, T.T. Branchflower, E.J. Ng, and T.W. Kenny, "Fatigue Experiments on Single Crystal Silicon in an Oxygen-Free Environment", Journal of Microelectromechanical Systems, 24, 2, (2015).

[9] W.W. Van Arsdell, and S.B. Brown, "Subcritical Crack Growth in Silicon MEMS", Journal of microelectromechanical systems, 8, 3, (1999).

[10] D.H. Alsem, R. Timmerman, B.L. Boyce, E.A. Stach, J.T.M. De Hosson, and R.O. Ritchie, "Very High-Cycle Fatigue
Failure in Micron-Scale Polycrystalline Silicon Films: Effects of Environment and Surface Oxide Thickness", Journal of Applied Physics, 101, 1, (2007).

[11] D.H. Alsem, C.L. Muhlstein, E.A. Stach, and R.O. Ritchie, "Further Considerations on the High-Cycle Fatigue of Micron-Scale Polycrystalline Silicon", Scripta Materialia, $59,9,(2008)$.

[12] J. Bagdahn, and W.N. Sharpe Jr, "Fatigue of Polycrystalline Silicon under Long-Term Cyclic Loading", Sensors and Actuators A: Physical, 103,1-2, (2003).

[13] C.L. Muhlstein, S.B. Brown, and R.O. Ritchie, "High-Cycle Fatigue and Durability of Polycrystalline Silicon Thin Films in Ambient Air." Sensors and Actuators A: Physical, 94, 3, (2001).

[14] I.B. Flader, Y. Chen, C. Ahn, D.D. Shin, A.L. Alter, J. Rodriguez, and T.W. Kenny, "Epitaxial Encapsulation of Fully Differential Electrodes and Large Transduction Gaps for MEMS Resonant Structures", IEEE 31st International Conference on Micro Electro Mechanical Systems (MEMS), Belfast, Ireland, 1/21 - 25/18, IEEE. (2018).

[15] Y. Chen, I.B. Flader, D.D. Shin, C.H. Ahn, and T.W. Kenny, "Robust Method of Fabricating Epitaxially Encapsulated MEMS Devices with Large Gaps", Journal of Microelectromechanical Systems, 26, 6, (2017).

[16] I.B. Flader, Y. Chen, D.D. Gerrard, and T.W. Kenny, "Wafer-Scale Encapsulation of Fully Differential Electrodes for Mutli-Axis Inertial Sensing", International Conference on Solid-State Sensors, Actuators, and Microsystems (Transducers), Kaohsiung, Taiwan, 6/18 - 22/17, IEEE, (2017), pp. 591-594.

[17] Y.B. Gianchandani, M. Shinn, and K. Najafi, "Impact of Long, High Temperature Anneals on Residual Stress in Polysilicon", International Conference on Solid-State Sensors and Actuators (Transducers), Chicago, IL, USA, 6/16 - 19/97, IEEE, (1997), pp. 623-624.

[18] H. Kahn, L. Chen, R. Ballarini, and A.H. Heuer, "Mechanical Fatigue of Polysilicon: Effects of Mean Stress and Stress Amplitude", Acta Materialia, 54, 3, (2006).

\section{CONTACT}

*A.L. Alter, tel: +1-651-808-3803; annealtr@stanford.edu 